\title{
Investigación educativa: competencias desarrolladas en un curso de prevención en abuso infantil ${ }^{*}$
}

\author{
Ana María Martorella \\ Profesionales Latinoamericanos contra el Abuso de Poder, Argentina \\ amartor@intramed.net
}

\section{RESUMEN}

El presente trabajo es el resultado de una investigación educativa, de tipo cualicuantitativa, de un curso semipresencial sobre prevención en abuso infantil que se dirigió a un grupo heterogéneo de profesionales comprometidos con la población infantil y adolescente durante ocho meses con encuentros mensuales. El rol docente estuvo a cargo de una médica pediatra especialista en psiquiatría infantojuvenil. Se implementaron diversas estrategias pedagógicas y se administraron contenidos conceptuales, procedimentales y actitudinales que favorecieran la adquisición de competencias adecuadas para el objetivo propuesto en el diseño curricular. Entre los resultados observados, durante las evaluaciones diagnóstica, formativa y final resaltan el interés

manifiesto por la resolución de la victimización infantil, la dificultad para el trabajo en equipo interdisciplinario y el abandono del curso por parte del $75 \%$ de los estudiantes inscriptos. Se puede concluir que este tipo de labor profesional demuestra la replicación de modelos identificatorios de roles estereotipados de género, culturalmente asignados a las mujeres en el cuidado de la infancia, y que la victimización infantil genera abandono de la tarea por resistencia al sufrimiento.

Palabras clave: abuso infantil y negligencia; competencias profesionales; estrategias pedagógicas; diseño curricular; contenidos conceptuales, procedimentales y actitudinales; niveles de prevención; trabajo interdisciplinario.

Cómo citar: Martorella, A. (2021). Investigación educativa: competencias desarrolladas en un curso de prevención en abuso infantil. Ciencias Sociales y Educación, 10(19), 113-140. https://doi.org/10.22395/ csye.v10n19a5 Recibido: 18 de junio de 2020.

Aprobado: 29 de julio de 2020. 


\section{Educational Research: Competences Developed in a Child Abuse Prevention Course}

\section{ABSTRACT}

This work is the result of a qualitativequantitative educational research, of a blended course on prevention of child abuse. It was aimed at a heterogeneous group of professionals committed to the child and adolescent population. It took place for eight months long with monthly meetings. The teaching role was in charge of a pediatrician specialist in child and adolescent psychiatry facility. Various pedagogical strategies were implemented through the administration of conceptual, procedural and attitudinal content, which favored the acquisition of suitable competences for the objective proposed in the curriculum design. The evident interest in the resolution of child victimiza- tion, the difficulty for interdisciplinary teamwork and the dropout of the course by $75 \%$ of the enrolled students stand out among the results observed during the diagnostic, formative and final evaluations. It can be concluded that this type of professional work shows the replication of identifying models of stereotyped gender roles culturally assigned to women in childcare, and that child victimization generates abandonment of the task due to resistance to suffering.

Keywords: child abuse and neglect; professional competences; pedagogical strategies; curricular design; conceptual, procedural and attitudinal content; prevention levels; interdisciplinary work.

\section{Pesquisa educacional: competências desenvolvidas em curso de prevenção de abuso infantil}

\section{RESUMO}

Este trabalho é o resultado de uma pesquisa educacional qualitativa-quantitativa, de um curso misto de prevenção de abuso e negligência infantil, destinado a um grupo heterogêneo de profissionais comprometidos com a população infantil e adolescente, durante oito meses com reuniões mensais. O papel de professor foi feito por um pediatra especialista em psiquiatria infantil e adolescente. Várias estratégias pedagógicas foram implementadas, por meio da administração de conteúdo conceitual, processual e atitudinal, o que favoreceu a aquisição de competências adequadas ao objetivo proposto no desenho curricular. Entre os resultados observados, durante as avaliações diagnósticas, formativas e finais, destacam-se o interesse evidente na resolução da vitimização infantil, a dificuldade para o trabalho em equipe interdisciplinar e o abandono do curso por $75 \%$ dos alunos matriculados. Conclui-se que esse tipo de trabalho profissional demonstra a replicação da identificação de modelos de papéis de gênero estereotipados, culturalmente atribuídos às mulheres na assistência à infância, e que a vitimização infantil gera abandono da tarefa devido à resistência ao sofrimento.

Palavras-chave: abuso e negligência infantil; habilidades profissionais; estratégias pedagógicas; Desenho curricular; conteúdo conceitual, processual e atitudinal; níveis de prevenção; trabalho interdisciplinar. 


\section{Introducción}

El presente trabajo se ocupa de realizar una investigación educativa a partir de la descripción, análisis y evaluación del desarrollo del curso Prevención de Abuso Infantil y Abandono, dictado por una médica pediatra y especialista en Psiquiatría Infantojuvenil, con amplio y prolongado entrenamiento en casos de abuso infantil y en pedagogía médica (Martorella, 2013b), cuyo rol docente corresponde al estilo democrático-directivo basado en el dominio de competencias. Dicho curso, de tipo semipresencial, se llevó a cabo a través de ocho encuentros mensuales durante un ciclo lectivo en la sede del Colegio de Médicos, en la ciudad de Mar del Plata, provincia de Buenos Aires. Estuvo dirigido a profesionales de diferentes disciplinas relacionadas con el trabajo con menores que se desempeñan en el ámbito judicial, educativo o sanitario. Este curso consistió en la transmisión de contenidos conceptuales, procedimentales y actitudinales pertinentes que se refieren a abuso infantil físico, emocional y sexual, así como sobre la negligencia en la legislación nacional e internacional sobre el tema. La metodología implementada se ha basado en la secuenciación de conocimientos progresivamente más complejos e integradores a partir de los indicadores diagnósticos y dinámicos del abuso físico, que atraviesa los del abuso emocional, para llegar al abuso sexual. De esta manera, se logra la adquisición de competencias necesarias para la elaboración de estrategias de intervención transdisciplinarias e interinstitucionales adecuadas. Los resultados observados durante el dictado del curso están relacionados con la continuidad por parte de los asistentes al mismo; el acuerdo de una sistematización metodológica en la presentación de la casuística, tanto en los trabajos prácticos, el trabajo grupal para la evaluación parcial y final, como en la evaluación permanente; y una encuesta anónima en el primer encuentro donde se comunica el pacto pedagógico (lugar y tiempo de encuentros, presentación de trabajos prácticos grupales, lectura de la bibliografía aportada en forma de cuatro módulos, defensa del trabajo final transdiciplinario, participación activa durante el desarrollo de los ateneos clínicos, consultas tutoriales a través de medios digitales), que permitió evaluar el logro de los objetivos propuestos al finalizar el curso.

Por lo tanto, la presente investigación educativa tiene por objetivo comprobar si las estrategias pedagógicas planteadas han facilitado el logro del perfil de egresado propuesto, a través de la adquisición de las competencias necesarias para la compleja labor profesional con niños, niñas y adolescentes víctimas de los diferentes tipos de abuso y negligencia.

\section{Marco teórico y conceptual}

A pesar de que en Argentina no existen datos estadísticos confiables ofrecidos por fuentes gubernamentales ni privadas, sobre el maltrato infantil y el abuso 
sexual infantil hay autores que han estudiado la prevalencia de estas problemáticas a nivel mundial. Entre ellos, Losada (2012) apunta:

$\mathrm{El}$ abuso sexual infantil es un problema frecuente en todas las sociedades y culturas. Sus efectos negativos muestran la gravedad e ilustran las necesidades profesionales de un mayor conocimiento al respecto. La incidencia y prevalencia del abuso sexual infantil, como conceptos estadísticos usados en epidemiología, aportan la distribución y evolución de la problemática en la población. (p. 202)

La misma autora aporta datos que remontan a los años cuarenta del siglo pasado sobre un estudio de Kinsey, quien, a través de encuestas a 4.441 mujeres estudiantes universitarias, obtuvo un reporte de al menos un episodio de abuso sexual antes de la adolescencia en el $24 \%$ de las encuestadas y que solo en el $49 \%$ de los casos el abusador era un desconocido (Losada, 2012). Por tal motivo, se considera de gran importancia la capacitación y formación en esta problemática a profesionales de diversas disciplinas relacionadas laboralmente con la minoridad.

Con el objeto de analizar los resultados observados al finalizar el curso, se deben conocer los elementos que intervienen en el proceso enseñanza. El aprendizaje incluye los siguientes contenidos: i) conceptuales (los diferentes tipos de abuso y maltrato que victimizan a los menores); ii) procedimentales (estrategias pedagógicas implementadas); y actitudinales (empatía y ética profesional). Estos contenidos tienden al logro del perfil profesional esperado, que se basa en la adquisición de las competencias requeridas para alcanzar una adecuada intervención profesional frente a esta problemática infantojuvenil. A continuación, se relacionan algunas definiciones sobre los tipos de maltrato y el abuso sexual infantil:

1) Maltrato físico: en 1961, Kempe expuso por primera vez el síndrome del niño golpeado como resultado de prácticas disciplinarias culturales. Por otro lado, Finkelhor y Korbin han sugerido que su definición debe incluir cualquier comportamiento proscripto y prevenible que cause daño y lesiones en los niños (Helfer, Kempe y Krugman, 1984).

2) Maltrato psicológico o emocional: es el resultado de conductas por acción o por omisión que pueden ocurrir en situaciones esporádicas o a través de un modelo crónico de interacción, bien sea por conductas sutiles o extremas, que provocan daño psíquico en los niños (Brassard y Hardy, 1984).

3) Abuso sexual infantil: Entre las varias definiciones propuestas, se han seleccionado las que expone Martorella (2010):

Helfer y Kempe (1976): Abuso Sexual es todo aquello que involucre a niños y adolescentes, dependientes y mentalmente inmaduros, en actividades sexuales 
que ellos no pueden comprender conscientemente, para las que ellos no poseen capacidad de autorización consciente, o que violan tabúes sociales en relación a los roles familiares.

National Center on Child Abuse \& Neglect (USA): Cualquier contacto u otra interacción entre un niño y un adulto, siendo el niño usado para estimular el placer sexual del adulto o cualquier otra persona. El abusador puede ser menor de 18 años, pudiendo ser considerado como abusador si existe una diferencia significativa de edad entre él y su víctima, o si el abusador presiona o controla los hechos y actitudes de la víctima. [...]

[En el] CIE 10, se lo considera dentro del eje V entre las situaciones psicosociales anómalas en su punto I: relaciones intrafamiliares". (p. 12)

Martorella (2010) refiere que, "[a] su vez se diferencia al abuso sexual, como acto ejercido por los responsables del cuidado del niño, del asalto sexual, considerado un acto sexual cometido por personas que no son responsables del cuidado del niño" (p. 12). Por otro lado, comenta que "los padres que abusan sexualmente a sus hijos pueden también asaltar a otros niños fuera de su familia, como también permitir que sus hijos sean abusados por otros" (Martorella, 2010, p. 12).

Otra forma de abuso sexual infantil es la victimización a los menores a través de la prostitución.

En este sentido, Martorella (2010) afirma que es importante comprender que "cuando los adultos tienen sexo con niños, el niño es siempre la víctima", y que, entre las formas de presentación del abuso sexual infantil (ASI), se incluyen "caricias en los genitales del niño; caricias por parte del niño en los genitales del agresor; coito; violación; sodomía; voyeurismo; estimulación genito-oral; estimulación verbal; exhibicionismo; explotación comercial a través de prostitución; la producción de material pornográfico" (p. 12) y la mutilación genital.

Por otro lado, dentro de estas consideraciones también es importante definir el abandono y la negligencia:

4) Abandono y negligencia: se define como la condición en la que un cuidador responsable del niño, bien sea deliberada o por extraordinaria desatención, permite que un niño experimente sufrimiento evitable o fracase en proveer recursos generalmente esenciales para su desarrollo y crecimiento (Helfer, Kempe y Krugman., 1984).

Para comprender los efectos de todos estos tipos de victimización infantojuvenil, es necesario conocer el desarrollo del sistema nervioso central (SNC) durante las diferentes etapas evolutivas, a través de los aportes actuales de las neurociencias. Estos aportes han permitido develar los procesos de neuroplasticidad y neurodesarrollo infantil que se ven afectados por las experiencias traumáticas, así 
como el trastorno por estrés postraumático que provoca la afectación de las habilidades cognitivas a nivel del proceso de encefalización, la corteza cerebral y de las interconexiones dendríticas y sinápticas cerebelosas en los primeros años de vida. Estas alteraciones se evidencian a través de anormalidades a nivel de estudios complementarios (RMN, EEG, potenciales evocados) sobre las características del estrés postraumático. Asimismo, se afecta el control límbico de los impulsos, lo que explica las conductas disruptivas y las dificultades en la regulación de las emociones, así como otros signos y síntomas diagnósticos (Martorella, 2015).

\section{Competencias}

Durante el proceso de enseñanza/aprendizaje, para alcanzar las expectativas de logro, se requiere que el docente posea tanto habilidades pedagógicas como conocimientos expertos en su disciplina. Esto quiere decir que requiere las competencias necesarias para promover la adquisición de contenidos y destrezas por parte del estudiante. Para poder comprender dichas competencias aplicadas al tema que nos ocupa en este artículo, debemos conocer las definiciones propuestas por diversos autores, que se señalan a continuación.

En el campo tecnológico aparecen definidas por primera vez las competencias en relacionadas con los procesos productivos. Varios autores han aportado diferentes elementos para conceptualizarlas, y se basan en diferentes supuestos previos con los que opera cada uno de ellos. El análisis de dichos elementos fundamentales se debe tener en cuenta al momento de elaborar el diseño curricular (Candreva, Martorella y Susacasa, 2011). A continuación, se citan diferentes definiciones sobre competencia laboral que se han desarrollado en el transcurso de los años:

- Un motivo, un rasgo, una habilidad, un aspecto de su imagen personal o de su rol social o un cuerpo de conocimientos puede ser una característica útil para el desempeño de un trabajo o profesión (Boyatzis, 1982). Esta definición tiene en cuenta la motivación, los rasgos personales, las habilidades, los conocimientos, etc., que se evidencian en la forma en que la persona se comporta, actúa, se desempeña, hace y se relaciona (Martorella y Candreva, 2011).

- Para Montmollin (1984), las competencias pueden ser puestas en práctica ante un nuevo aprendizaje a través de saberes y saber-hacer estabilizados, de conductas adecuadas, razonamientos y procedimientos pertinentes.

- Por otro lado, se la puede definir como "la capacidad individual para aprender actividades que requieran una planificación, ejecución y control autónomos" (Candreva et al, 2011).

- Hayes (1985) considera que es aquella capacidad que permite alcanzar un objetivo mediante el uso del conocimiento y las destrezas relacionadas con productos y procesos. 
- Jessup (1991) la define como el conjunto específico de destrezas necesarias para desarrollar un trabajo mediante la aplicación de las cualidades necesarias para desempeñar un rol profesional o laboral.

- Gilbert y Parlier (1992) las definen como los conocimientos, las capacidades de acción y los comportamientos estructurados necesarios para alcanzar un objetivo específico en una situación dada.

- Bunk (1994) considera que, para ejercer una profesión y resolver problemas de forma automática, pero al mismo tiempo flexible para colaborar con su entorno en la organización laboral, se requiere la adquisición de un conjunto de conocimientos, destrezas y aptitudes pertinentes a dicha tarea.

- Le Boterf aporta varias definiciones en diferentes momentos. Así en 1994, dice que la competencia se construye a partir de un saber actuar, pero requiere capacidad y deseo (Le Boterf, 1994). Mientras tanto, en 1997, refiere que las competencias movilizan, integran y orquestan recursos, en la forma de saber actuar, saber hacer o tener las actitudes pertinentes de acuerdo con la singularidad de cada situación, en analogía con otras experiencias conocidas (Le Boterf, 1997). En su obra publicada en el año 2000, la define como una construcción resultante de una combinación pertinente de varios recursos, tales como conocimientos, redes de información, redes de relación, saber hacer (Le Boterf, 2000).

- Bélisle y Linard (1996) consideran que la asimilación de información y de experiencia facilitan la adquisición de habilidades, mediante una capacidad para realizar una tarea profesional que cumpla con criterios estándares de rendimiento en condiciones específicas.

- La competencia no está en los saberes en sí mismos, sino en el conjunto de conocimientos y saber-hacer a partir de la utilización de los recursos del ambiente (Ginisty, 1997).

- La competencia se manifiesta a través de la movilización de diversos recursos cognitivos frente a situaciones específicas (Perrenoud, 2000).

- También se la ha definido como aquellas actitudes, destrezas, habilidades y conocimientos necesarios para ejecutar adecuadamente las funciones productivas requeridas en la realización de un trabajo (Candreva, Martorella y Susacasa, 2011).

- La Comisión de la Función Pública del Canadá las describe como los conocimientos, capacidades, habilidades y comportamientos manifiestos por un empleado en el desempeño de su labor, considerados factores de influencia positiva para el logro de los resultados pertinentes (Gobierno de Canadá, 2007).

Fernández (1997) se refiere al componente "movilizador" de la competencia cuando afirma que solo es definible en la acción, por lo que no puede reducirse solo al saber o al saber-hacer. Su adquisición no depende solo de la formación ni de la capacidad de cumplir instrucciones. Además, se valoran las reacciones, decisiones y conductas que se aplican durante la labor. Su adquisición y desarrollo es dinámico y dependiente del contexto. La competencia "está en la 
cabeza del individuo, es parte de su acervo y su capital intelectual y humano" (Candreva, Martorella y Susacasa, 2011, p. 4).

Para Malpica (1996), el eje principal de la educación por competencias es el desempeño, es decir, cómo el individuo realiza una actividad y administra los recursos y su saber-hacer más allá de sus conocimientos. En este sentido, afirman Candreva, Martorella y Susacasa (2011):

Desde esta perspectiva, lo importante no es la posesión de determinados conocimientos, sino el uso que se haga de ellos. Este criterio obliga a las instituciones educativas a replantear lo que comúnmente han considerado como formación. Bajo esta óptica, para determinar si un individuo es competente o no lo es, deben tomarse en cuenta las condiciones reales en las que el desempeño tiene sentido, en lugar del cumplimiento formal de una serie de objetivos de aprendizaje que en ocasiones no tienen relación con el contexto.

\section{$[\ldots]$}

La formación basada en competencias presenta varias diferencias con la formación, que se clasifica como convencional o tradicional, pero no existe duda de que la diferencia más clara y definitiva es que tiene como referente una competencia y esto obliga a que su diseño curricular se ordene desde el comienzo en torno a su desempeño. El diseño curricular por competencias responde a las necesidades de nuestros profesionales, así como a los cambios de los contextos. Los individuos formados en el modelo de competencias profesionales reciben una preparación que les permite responder de forma integral a los problemas que se les presenten con la capacidad de incorporarse más fácilmente a procesos permanentes de actualización, independientemente del lugar en donde se desempeñen. Otro aspecto importante, con relación a este modelo, estriba en el desarrollo de las capacidades de pensamiento y reflexión, para la identificación, así como la toma de decisiones en situaciones problemáticas no contempladas durante la formación. La formación por competencias implica una preparación más completa, integral y flexible, que permite dar respuesta a las necesidades de los individuos, de la comunidad y de la sociedad teniendo en cuenta los diferentes contextos y culturas.

\section{$[\ldots]$}

El concepto de competencia otorga un significado de unidad e implica que los elementos del conocimiento tienen sentido sólo en función de conjunto y la capacidad, que tiene el ser humano de integrar y movilizar sistemas de conocimientos, habilidades, hábitos, actitudes y valores para la solución exitosa de aquellas actividades vinculadas a la satisfacción de sus necesidades cognitivas y profesionales. Los procesos de formación basada en competencias son bastante nuevos y parecen surgir, principalmente en la necesidad del trabajador/a de obtener un servicio de formación, para superar un resultado de evaluación, y en los procesos de modernización de los sistemas de formación, que ven en el movimiento de las competencias un referente muy válido para optimizar los insumos del diseño curricular y organizar el proceso enseñanza-aprendizaje entorno a la construcción de capacidades que favorezcan un desempeño exitoso. (pp. 5-9) 


\section{Investigación educativa}

Como la educación es considerada una ciencia fáctica, dedicada a promover modificaciones conductuales e intelectuales, merece ser investigada y evaluada en su metodología y efectos. De esta manera, se pueden conocer y medir sus resultados, sean positivos o negativos, que nos permitan decidir replicar o evitar las estrategias pedagógicas aplicadas durante el proceso de enseñanza/aprendizaje que nos ocupa. Para tal fin, en este apartado se hará una reseña acerca de los objetivos y métodos de investigación educativa, que fundamentan el presente estudio. Este análisis tiene por objeto facilitar la reproducción futura del presente proyecto de capacitación y entrenamiento, en diferentes contextos, por otros profesionales interesados en la prevención del abuso infantil.

Los rápidos cambios sociales y tecnológicos exigen la construcción de nuevas imágenes de la práctica social en general y de las empresas en particular. Se piensa que la indagación realizada por los entes de estas instituciones puede constituirse en una de las herramientas de cambio y mejora en la calidad organizacional. En el ámbito educativo, cualquier profesional de la docencia vinculado al mundo de las instituciones, está llamado a desempeñar un papel clave como investigador de su propia práctica, con la finalidad de mejorar su formación, su desempeño en el aula, escuela y comunidad, en la búsqueda de un cambio organizacional hacia una transformación sociocultural. (González et al., 2007, p. 280)

Para estos autores, las organizaciones deben basar la investigación en un eje central colaborativo, cuyos participantes, pasantes y profesores deben ocupar el papel de promotores pedagógicos e investigadores sobre la práctica docente para mejorar la práctica de la enseñanza. Sus investigaciones deben realizarse desde y para las organizaciones educativas, con sentido en el entorno de los problemas escolares, y constituirse en una excelente herramienta para mejorar la calidad de dichas organizaciones. Ellos refieren, además, que la investigación es una actividad humana que cumple una función muy importante para la sociedad. Es una actividad cuyo objetivo es alcanzado mediante el conocimiento de los hechos, las causas, las relaciones y las consecuencias en cada fase del proceso, con plena conciencia de todos sus elementos y factores, para lograr su eficacia. La curiosidad, la reflexión, el cuestionamiento y la duda son bases fundamentales de toda genuina investigación. Esta será educativa si favorece que todos los involucrados desarrollen nuevas alternativas de comprensión que le permitan emprender acciones propias, autónomas y compartidas sobre el sentido de la práctica y sus mejoras. En el contexto educativo, la investigación es un encuentro entre personas con una actividad ética en permanente reflexión y cuestionamiento. 
La investigación no puede reducirse a una actividad técnica, debido a la profundidad del proceso. Es una actividad cuyos protagonistas son los docentes, los estudiantes y la comunidad donde se desarrollan, en la cual el docente actúa con el sujeto en forma integral y teniendo en cuenta su comportamiento influenciado por sus creencias, actitudes, costumbres y contexto. Mientras tanto, el alumno procesa la información recibida a partir de sus experiencias previas, costumbres y contexto histórico. Esto hace que el proceso de aprendizaje sea una situación incierta, única, cambiante y compleja que presenta conflicto de valores al definir las metas y seleccionar los medios para alcanzarlas. El docente interviene en un medio escolar comunitario complejo, psico-social-espiritual, vivo y cambiante que surge de la interacción simultánea de múltiples factores y condiciones (sociales, económicos, culturales, políticos, etc.). Su habilidad para manejar la compleja realidad y resolver situaciones conflictivas determinará el éxito de su accionar, lo cual requiere "un proceso de reflexión en la acción o una conversación reflexiva con la situación problemática concreta, que permitirá crear nuevas realidades, corregir e inventar" (González et al., 2007, p. 281).

González et al. (2007) consideran que la reflexión ante los conflictos conduce a una forma de actuar inteligente y creativa. A su vez, refieren que también se reflexiona durante y sobre la acción para su análisis posterior, e incluyen un tipo de reflexión sobre la reflexión en la acción para someterla a un cuestionamiento más profundo. Esta reflexión se constituye como un instrumento óptimo para alcanzar un aprendizaje significativo y para un genuino desarrollo profesional que permita iniciar el camino hacia el cambio. Investigando en y sobre su acción, el docente se transforma en un investigador educativo en el ámbito escolar y contextual que depende de sus propios descubrimientos y de la teoría elaborada en su labor pedagógica a través de su reflexión permanentemente. La construcción y aplicación de su propia teoría le permiten observar su resultado positivo o negativo, para reflexionar, corregir y reconstruir nuevamente. Si su interés investigador es genuino, entonces le importan más la comprensión de fenómenos y procesos que la acumulación de datos. De esta manera, se asume el método dialéctico de investigación reflexión-acción a partir de la experiencia, para someter los resultados y su teoría al cuestionamiento y al proceso de reelaboración.

Al iniciar toda investigación educativa, en y sobre el rol docente y el proceso de enseñanza-aprendizaje, se deben fijar objetivos claros de sensibilización y concientización de las problemáticas mediante la reflexión aguda, la atención puesta en aspectos importantes y pertinentes a dicha tarea, la clarificación de los problemas y el estímulo al debate e intercambio de opiniones. La finalidad de esta es favorecer la comprensión profunda, la flexibilidad y la adaptación, que ayudan a facilitar 
habilidades y destrezas necesarias para la resolución de conflictos. Toda investigación supone la incesante búsqueda de la verdad, el desentrañar lo aparente, la precisión, el rigor y objetividad en el conocimiento, la exploración, la creatividad, la imaginación, la duda constante, la actitud crítica; la formulación permanente de por qué, la búsqueda de explicaciones para todas las cosas, la autodisciplina, la perseverancia y el trabajo metódico, en un ambiente que fomente: curiosidad, búsqueda, experimentación y modos naturales de aprender. Por esto, es urgente que el docente logre estimular en los alumnos la curiosidad de saber, preguntar, explorar, comprobar, experimentar, perfeccionar, aprender por deseo, no por miedo u obligación; fomentar en ellos el sano hábito de dudar, enseñarles a construir, formular y expresar con libertad sus preguntas; ayudarles a razonar, comprender, argumentar, defender su punto de vista, aceptar y respetar posturas diferentes, a ver "la cosa" desde diversos ángulos. Se debe combatir la memorización mecánica a favor de un aprendizaje significativo, basado en la comprensión, razonamiento, explicación y descripción de los hechos. (Gonzáles et al., 2007, pp. 282-283)

Para el logro de esta propuesta, se requiere generar la necesidad de la lectura comprensiva y crítica, así como la escritura sistemática, a partir de los métodos de aprendizaje que permiten aprender haciendo, comprobando y experimentado. Además, se debe fortalecer en el alumno la autoestima basada en la autoexigencia y la tolerancia a la frustración. La tarea de investigación apropiada por parte del estudiante se verá garantizada si el educador posee experiencias previas sobre el acto de investigar genuino y tiene el deseo de transmitirle la necesidad de desarrollar actitudes requeridas para tal fin, como ser la apertura a la discusión, la profundización de los temas de estudio, la búsqueda de soluciones e innovaciones y el procesamiento de conocimientos previos. Esto quiere decir que practica dichas actitudes epistemológicamente, tanto en su cotidianeidad como en su experiencia educativa, con el objeto de conocer la realidad y de adquirir nuevos aprendizajes. Por lo tanto, el docente debe poseer formación sobre los tipos de investigación y los procesos de aprendizaje basados en la investigación-acción y en la reflexión sobre su práctica (González et al., 2007).

Como todo proceso investigativo corresponde a un acto científico, se debe conocer la definición de ciencia y sus propósitos. El objetivo de la ciencia es elaborar teorías válidas, que resuelvan problemas. Para Laudan (1986), dichos problemas se dividen en dos tipos:

- Empíricos: exigencias correspondentistas. Se resuelve con la teoría que se deduce de un enunciado del problema y resuelve o elimina un problema conceptual. El valor de una teoría depende del número de problemas que resuelva.

- Conceptuales: exigencias coherentistas. (Citado en Martorella, 2013a, p. 3)

Por otro lado, Hume (1984) afirma: 
El conocimiento surge de toda acción racional, no se relaciona con hechos singulares o aislados, indica correlaciones, ligaduras y pautas que gobiernan la estructura de lo real y que son expresadas por algunas hipótesis. Es un presupuesto para la transformación de la realidad. Se produce en relación con factores sociales, políticos, económicos, psicológicos, ideológicos, y se valida por la vinculación con la justificación del conocimiento, con primacía lógico lingüística. (Citado en Martorella, 2013a, p. 3)

En el proceso de investigación se aplica el método hipotético-deductivo, que permite el contraste de hipótesis a través de los resultados observados, con el objeto de determinar la falsedad de una proposición, pero no su veracidad. El contraste de las teorías, mediante sus consecuencias empíricas, permite que sean corroboradas o descartadas cuando son refutadas por la falsedad de alguna de sus consecuencias. De este modo, las teorías con sectores teóricos puros se denominan hipótesis interpretativas. Estas surgen por introducción de reglas de correspondencia y enunciados mixtos o teóricos observacionales para facilitar la vinculación con enunciados empíricos básicos para que ocurra la contrastación. Esto quiere decir que se trata de descubrir una realidad específica de hechos en cada área del conocimiento por medio de la hipótesis (Martorella, 2013a).

Cuando nos referimos a los datos obtenidos en la vida cotidiana a través de la observación directa, entonces se constituye la base empírica epistemológica o un tipo de información que se obtiene sin mediatez científica ni auxilio de instrumentos, considerada teoría científica o argumento interno para la ciencia, que son aceptadas por la comunidad científica con el apoyo de lenguaje ordinario (Klimovsky, 1994). Mientras tanto, la observación de los objetos constituye la base empírica metodológica, que conduce a los científicos a la aceptación de las teorías.

Esto quiere decir, según Klimovsky (1994), que:

Toda investigación requiere de un método insertado en la realidad problematizada; debe ser conducida por las hipótesis; detecta alguna dificultad, en una situación práctica o teórica, que guía la búsqueda de algún orden entre los hechos para solucionarla; requiere de un problema, que es ocasión para la investigación; busca una solución que es el objetivo de la investigación; y requiere conocimiento y relevancia (Citado en Martorella, 2013a, p. 12-13).

\section{Material y método}

El diseño mixto de esta investigación educativa surgió como necesidad de comprender los resultados observados en el curso de capacitación considerado en el presente estudio. A partir de la indagación y mediante la recolección de datos cualitativos acerca de la conformación del grupo de estudiantes, se llevó a cabo un análisis cuantitativo basado en el lugar de desempeño laboral y en la formación profesional de cada uno de los inscriptos que permitiera una descripción 
estadística de la población en estudio. Dichos datos se obtuvieron de una encuesta inicial, durante la primera clase del curso, con el objeto de describir las características heterogéneas del grupo participante.

Asimismo, en el mismo momento, los asistentes fueron consultados, de forma verbal y a modo de presentación con el resto de los participantes, sobre sus motivaciones para realizar el entrenamiento en la problemática de abuso infantil, lo cual pudiera aportar información al diagnóstico situacional de sus necesidades y carencias formativas profesionales. Esta reseña ha sido importante para cumplir los objetivos del presente trabajo. A través de la descripción de las características individuales y la exploración de las competencias previamente adquiridas, se posibilitó la interpretación concurrente de los resultados obtenidos y la elaboración de conclusiones a partir de los mismos. Así, los resultados obtenidos dan cuenta de los diferentes puntos de vista, representaciones, conceptos mentales, ideas, percepciones y significados de la misma realidad de acuerdo con las valoraciones subjetivas de los participantes.

Esto quiere decir que, a través de la manipulación observacional del objeto de estudio, se han aprovechado los datos recolectados para cumplir el propósito de evaluar las competencias adquiridas por los asistentes al finalizar el entrenamiento profesional. Estos datos han facilitado la medición longitudinal de los resultados observados y elaborar una perspectiva prospectiva de las competencias adquiridas por los participantes.

Por otro lado, a medida que se avanzaba en el desarrollo de las clases, los asistentes realizaban comentarios acerca de las características de trabajo en sus instituciones. En este sentido, describieron las dificultades y obstáculos con los que se encontraron ante cada caso particular de vulneración de derechos infantiles. Esta información también ha sido útil para estimar los beneficios en la adquisición de competencias que pudieran favorecer la mejor resolución de conflictos institucionales y de los casos de abuso infantil referidos por los estudiantes.

Los profesionales asistentes al curso se desempeñaban en diferentes instituciones públicas y privadas de ámbitos judiciales, educativos y sanitarios, donde recibían asiduamente casos de abuso infantil. Sin embargo, la institución no les brindaba capacitación y entrenamiento sobre las características particulares del abuso infantil y la negligencia, como tampoco para la adquisición de destrezas y habilidades que facilitaran la resolución de problemas, por lo que debían buscarla fuera de sus ámbitos laborales.

En su inicio, al curso concurrieron veintinueve profesionales, que actuaban tanto en el ámbito público (veinticinco) como en el privado (cinco), de los cuales uno se desempeñaba en ambas áreas. Con respecto al ámbito público, siete se 
desempeñaban en justicia, nueve en educación y nueve en salud. Por otro lado, al ámbito privado correspondían dos profesionales en justicia, dos en educación y uno en salud (figura 1). Entre las profesiones encontramos: doce trabajadores sociales (tres en poder judicial, cuatro en educación y cinco en sanidad); cuatro psicólogos, todos en el ámbito de la educación; tres docentes en educación; tres abogados en justicia; tres estudiantes; dos psiquiatras en justicia y sanidad; un terapista ocupacional; una enfermera; y una médica pediatra en sanidad (figura 2).

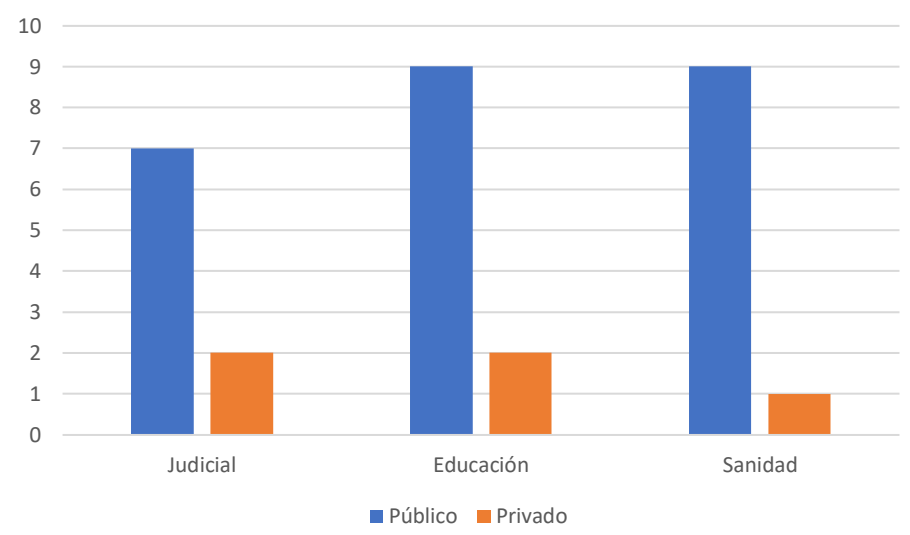

Figura 1. Origen laboral de los veintinueve estudiantes

Fuente: elaboración propia.

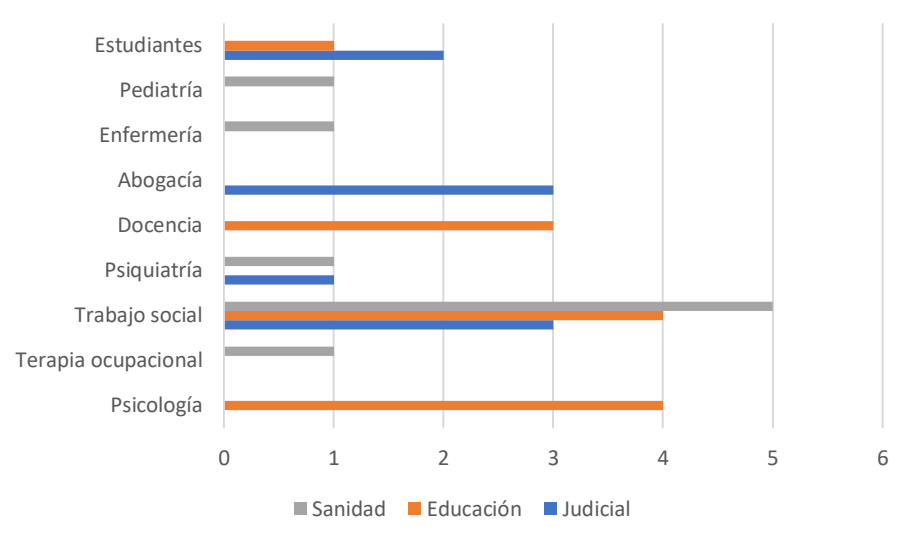

Figura 2. Profesiones de los estudiantes según su origen laboral Fuente: elaboración propia.

Durante los siete encuentros, en relación con las estrategias pedagógicas y las tecnologías didácticas empleadas durante las clases expositivas, se utilizaron diapositivas en seis encuentros, videos en tres y filminas en uno (figura 
3). Esto se hizo con el objetivo de favorecer los aprendizajes específicos de los temas programados para sistematizar, no solo la información teórica, sino también para orientar al estudiante en el objeto de estudio complementado con la experiencia personal de la profesional a cargo de la orientación de las clases.

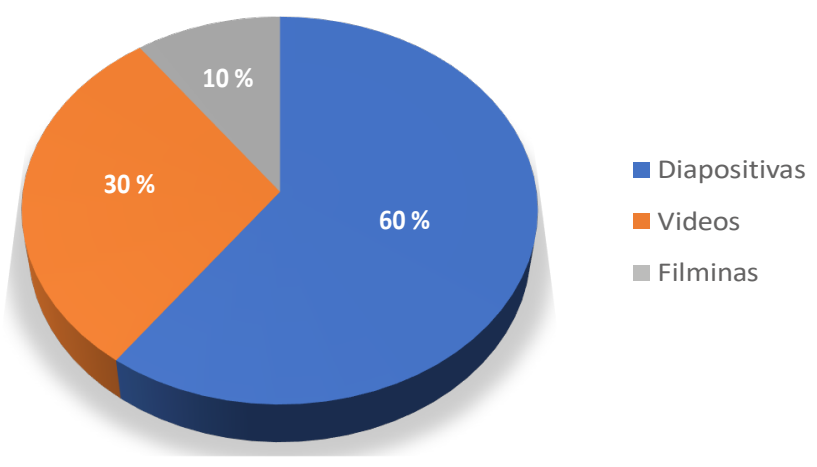

Figura 3. Tecnologías didácticas

Fuente: elaboración propia.

Con respecto a la didáctica, se tuvieron en cuenta las siguientes técnicas y estrategias pedagógicas:

- Proyección de diapositivas y filminas.

- Proyección de video-filmaciones.

- Exposición sistemática de la información.

- Presentación de casuística.

- Supervisión clínica y asesoramiento profesional especializados de la casuística presentada.

- Taller de reflexión.

- Discusión y debate.

- Orientación bibliográfica.

- Distribución de material impreso y textos de autores nacionales e internacionales.

- Ateneo bibliográfico.

- Registro fotográfico de la casuística presentada y del contexto al que pertenece. 
Con respecto a la metodología, se dedicaron dos encuentros y medio al tema del abuso físico, un encuentro y medio para abuso emocional, dos encuentros y medio para abuso sexual, un encuentro y medio para estrategias de intervención junto a niveles de prevención y legislación (figura 4).

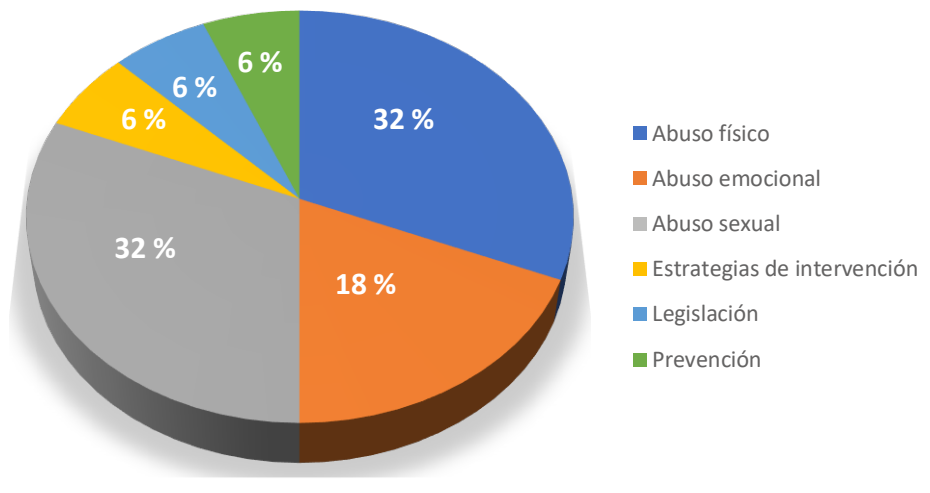

Figura 4. Tiempo en horas de dedicación por temas

Fuente: elaboración propia.

La elección de dicha secuenciación de actividades y contenidos se basó en la experiencia de la docente en relación con su propio proceso de aprendizaje de los diferentes aspectos de esta problemática, así como su labor profesional con menores que fueron víctimas de diferentes tipos de abusos y malos tratos. Esto se debe a que el abuso sexual infantil es el más complejo de los tres abusos, ya que en él se presentan conductas del agresor que afectan tanto el cuerpo como las emociones de la víctima infantil o adolescente, además de las regiones sensitivas erógenas. Finalmente, tanto su cuerpo como su aparato psíquico queda lesionado de un modo integral y potenciado. Esto quiere decir que las diferentes etapas del proceso de desensibilización del menor elegido como víctima se inicia en el contacto físico progresivo acompañado de un discurso que tiende a lograr la confianza del niño, niña y adolescente (NNA), para luego transformarlas en amenazas y frases culposas y vergonzosas que liman la autoestima de la víctima. Por lo tanto, el proceso de aprendizaje de comprensión de estos hechos aberrantes y sus efectos en las víctimas debería seguir las mismas instancias de aprendizaje para el caso de los profesionales comprometidos en su labor con infancias vulnerables.

Para ello, se propusieron los siguientes contenidos conceptuales, procedimentales y actitudinales en el currículo: 


\section{A. Contenidos conceptuales}

1. Conceptos de abuso infantil y negligencia.

2. Clasificación de abuso infantil:
a) Abuso físico.
b) Maltrato emocional.
c) Descuido físico.
d) Abuso sexual.

3. Indicadores diagnósticos de los diferentes tipos de abuso infantil.

4. Diagnóstico diferencial.

5. Diagnóstico de situación.

6. Abuso infantil y discapacidad: experiencias pedagógicas a través de talleres lúdicos adaptados a las necesidades especiales de los alumnos que presentaban retardo mental leve y moderado (Martorella y Portugués, 1998; Martorella, 2014a).

7. Estrategias de intervención clínica.

8. Estrategias de intervención social.

9. Estrategias de intervención pedagógica. Se ejemplificó con material fotográfico que fue producto de experiencias en educación sexual con alumnos de diversos niveles educativos y desarrollo cognitivo. Dicho material mostró técnicas pedagógicas lúdicas plásticas, que permitieron representar las diferencias genitales de los sexos a través del dibujo y de la manipulación de cerámica, durante la etapa diagnóstica inicial de saberes previos de los alumnos púberes en diferentes establecimientos educativos (Martorella, 2010; Martorella, 2013b; Martorella, 2014b; Martorella, 2015).

10. Estrategias de intervención jurídica.

11. Niveles de prevención (primaria, secundaria, terciaria).

\section{B. Contenidos procedimentales}

1. Elaboración de informes de casos comunicables.

2. Confección e interpretación de historias clínicas (anamnesis: semiología, epicrisis), legajos escolares e informe socioambiental.

3. Actuaciones legales (pericias, carátulas, expedientes). 
4. Modalidad de trabajo transdiciplinario e interinstitucional en redes.

5. Intervenciones clínico-educativo-judiciales (tratamiento médico psiquiátrico, pediátrico, psicológico, psicopedagógico, familiar).

6. Investigación en situaciones de riesgo.

7. Intervenciones en los diferentes niveles de prevención.

\section{Contenidos actitudinales}

1. Valoración de la salud física y psíquica del niño por parte de todos aquellos que trabajan con menores.

2. Responsabilidad en la ejecución de conductas y actos por parte de los adultos en relación con los niños y adolescentes.

3. Comprensión del desarrollo evolutivo infantil y la incidencia de las conductas abusivas y negligentes en el desarrollo físico y emocional de los menores.

4. Valoración del aspecto social de las actitudes violentas de los adultos frente a los menores.

5. Empatía.

En relación con la adquisición de competencias, se propusieron:

\section{A. Capacidades intelectuales}

Conocer el marco teórico de los elementos que intervienen en las situaciones de abuso infantil y negligencia.

\section{B. Capacidades prácticas:}

Aplicación de las competencias intelectuales para la elaboración de diagnósticos y estrategias de intervención clínica, pedagógica, social y jurídica, así como de actividades que correspondan a los distintos niveles de prevención.

\section{Capacidades sociales}

1. Respeto de los derechos del niño.

2. Actitud preventiva durante la tarea educativa (por ejemplo, educación sexual, diagnóstico de indicadores gráficos y de conductuales), sanitaria y judicial.

3. Establecimiento de redes solidarias entre los diversos sectores que interactúan con niños y adolescentes. 
4. Compromiso frente al desarrollo y crecimiento de los menores, así como a su contexto social y familiar.

5. Acuerdo en conductas no violentas frente y hacia los menores.

6. Valoración de los aportes que el menor hace a la sociedad (creatividad, espontaneidad, solidaridad, etc.).

A medida que se avanzaba en el desarrollo de los temas, secuenciados progresivamente según su grado de complejidad, se solicitó la elaboración de trabajos prácticos grupales que, inicialmente, presentaron un caso de abuso físico infantil (diagnóstico e intervención). Luego se indicó la necesidad de reelaborarlo con el agregado de los indicadores de abuso emocional, para posteriormente investigar los datos sugestivos de abuso sexual y las estrategias de intervención transdisciplinaria e interinstitucional.

Todo el proceso de enseñanza-aprendizaje se basó en diversas teorías del aprendizaje, como la del aprendizaje social, a través del modelado identificatorio y la del aprendizaje significativo de Ausubel, entre otras, con el objeto de facilitar el desarrollo cognitivo de los nuevos saberes (figura 1).
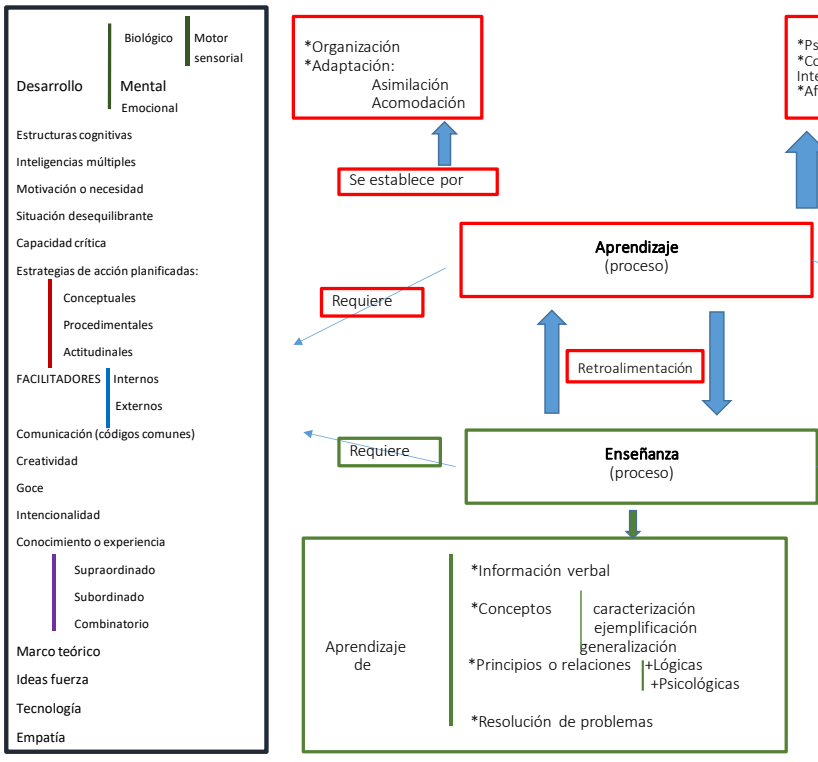

Figura 5. Proceso enseñanza- aprendizaje

Fuente: elaboración propia. 


\section{Resultados}

En este apartado se describen los resultados de las evaluaciones inicial diagnóstica, la formativa y la final en relación con la respuesta de los estudiantes y las competencias desarrolladas con respecto a los objetivos y a los contenidos propuestos junto a la autoevaluación del rol docente.

Sainz Leyva (2002) considera la evaluación del aprendizaje como una importante función imprescindible para que todo docente tenga un desempeño eficiente, aunque se comprueban diferentes dificultades que pueden afectar su calidad, lo que se constituye en uno de los espacios más frecuentes de enfrentamiento entre docentes y estudiantes. Para comprender el valor de aplicabilidad de la etapa evaluativa, resulta de interés la siguiente reflexión de esta autora cuando expresa:

El carácter no democrático e impositivo del estilo pedagógico de comunicación, que se pone en evidencia constantemente en el propio acto evaluativo y que, sin dudas, es una representación de la "violencia" escolar si entendemos por violento toda aquella imposición a la que son sometidos los educandos, sin que puedan cambiar fácilmente dicha situación. Por ello, la investigación de la evaluación del aprendizaje resulta un tema vital en el estudio de cualquier forma de violencia espiritual, que puede ocurrir en el ámbito educativo, y son necesarias las correcciones pertinentes para evitarlo. (Sainz Leyva, 2002, pp. 1-2)

Por tal razón, se realizan evaluaciones en diferentes momentos del curso que motiva este trabajo de investigación.

En el primer encuentro se promovió la presentación de cada uno de los concurrentes al curso con el objeto de realizar una evaluación diagnóstica inicial acerca de los conceptos y experiencias individuales de cada uno de los profesionales sobre esta problemática infantil, junto con las expectativas con respecto a los objetivos del curso, a modo de elaborar una planificación curricular adecuada a la heterogeneidad del grupo y sus necesidades. Dicha evaluación se llevó a cabo a través de una encuesta que indagó por los datos formativos y laborales, así como las expectativas y motivaciones con respecto al presente curso.

Como respuestas a resaltar de dicha presentación, surgieron claras manifestaciones sobre el interés personal, la movilización surgida a partir de la propia experiencia, la ansiedad por el desarrollo de los niños víctimas, la necesidad de ayudar y complementar conocimientos, el maltrato surgido de las crisis institucionales y la consiguiente negación de sus responsabilidades, la escasa comprensión de otros profesionales involucrados en esta problemática, el aumento de casos de abuso de menores, la necesidad de capacitación sobre pautas de crianza, el escaso compromiso sanitario y judicial, la necesidad de elevar el número de profesionales que trabajen con menores, el reconocimiento expreso sobre la necesaria formación 
profesional continua, la liberación de los sentimientos de culpa frente a la impotencia, el fomento del intercambio de experiencias en estrategias de intervención y articulación de las acciones, el reconocimiento de ausencia de capacitación previa, experiencias previas con adictos abusadores y abusados, el deseo de mejorar la calidad profesional en relación con el diagnóstico y las intervenciones, el requisito de aumentar el número de herramientas con el objeto de fortalecerse profesionalmente con respecto a los abordajes en situaciones de infancias vulnerables.

Con respecto a la evaluación formativa, esta se basó en la presentación grupal de la casuística: un caso fue presentado en forma completa y sistematizada, a la vez que los restantes se hicieron en forma asistemática. Por otro lado, se elaboraron cuatro trabajos prácticos de forma grupal, a la vez que dos participantes los desarrollaron en forma individual. Entre los trabajos prácticos individuales, una de las profesionales no logró comprender la consigna. Mientras tanto, entre los trabajos prácticos grupales, uno no cumplió la expectativa. Un trabajo individual y los grupales, excepto uno, no cumplieron con un orden metodológico, lo que comprobó ausencia de capacitación previa en metodología de la investigación. Otro individual fracasó en el desempeño profesional y en el trabajo transdisciplinario.

En la devolución de los trabajos prácticos, dos grupos no tuvieron continuidad. Dos trabajos grupales y uno individual demostraron buena receptividad de los señalamientos y satisfacción profesional por el enriquecimiento teórico del tema, que redundaba en beneficio individual y grupal.

En relación con la dinámica grupal, se observó actitud de escucha, aportes e intercambio de ideas, tanto entre los participantes entre sí como con la docente a cargo.

También cabe destacar la empatía con el sufrimiento del niño víctima, que permitía la comprensión de los sentimientos de venganza y culpa del niño abusado.

Se reflexionó sobre la necesidad de entrenamiento específico, que favoreciera el reconocimiento del abuso infantil y las correspondientes intervenciones adecuadas.

Con respecto a la dinámica familiar de los casos aportados, se destacó la inmadurez parental familiar como parte del diagnóstico. Se sugirió el aprovechamiento de los recursos humanos, a partir del aumento de la sensibilidad, a través de la percepción de los casos y de los niveles de comunicación. También, se cuestionó la responsabilidad profesional de los actores (profesionales). 
Se comprobaron sentimientos de angustia y frustración manifiestos en aquellos estudiantes que continuaron su asistencia al curso, la cual era contenida por el macrogrupo y sublimada a través de la búsqueda creativa de estrategias de intervención innovadora.

La frustración fue explicada como consecuencia directa de la falta de respuesta y seguimiento adecuado por parte de la institución en la que trabajaban y de otras instituciones intervinientes.

Avanzado el curso, surgió la necesidad grupal de ponerse de acuerdo sobre las competencias adecuadas de crianza y cómo trabajar sobre las funciones maternales positivas frente a las conductas parentales negligentes, de abandono o abusivas.

Se comprobó, de forma expresa, la disconformidad general por la legislación nacional y provincial vigente, así como la ausencia de contención social y económica a las víctimas y sus familias por parte del Estado y sus instituciones responsables.

En varias oportunidades, surgió el agradecimiento espontáneo verbal a la docente como única profesional que comprendía sus necesidades e inquietudes y respondía a las demandas emocionales de cada alumno más allá de su rol docente. La docente aprovechó su formación profesional en psiquiatría y salud mental, que requiere un notable desarrollo de empatía.

En el sexto encuentro se realizó la devolución a través de los señalamientos, que incluyeron: omitir la revelación de la identidad de la víctima o cualquier dato identificatorio; que el desarrollo no fuera telegráfico; que permitiera una lectura clara y que no confundiera al lector lego. Se sugirieron dos normativas de presentación de trabajos para alentar una organización metodológica de estudio e investigación que fuera comunicable.

En el primer modelo (anamnesis) se sugirió un ordenamiento que partiera del motivo de consulta, seguido de una metodología diagnóstica que utilizara pasos semiológicos que tendiera a caracterizar el abuso, sus lesiones y a identificar los indicadores del mismo. En el siguiente punto se solicitó aportar datos de la historia personal y escolar de la víctima (historia evolutiva, antecedentes comórbidos). Otro dato por investigar sería la dinámica familiar en relación con el funcionamiento de los roles junto a los datos socioeconómicos y culturales (la ficha o informe socioambiental). También se debían evaluar los factores de riesgo para el menor en cuestión y, si hubo revelación del abuso, determinar a quién y el modo. A partir de todo este desarrollo, se propuso describir las estrategias de intervención y de tratamiento educativo, judicial y sanitario, con un posterior seguimiento de la evolución terapéutica, sin olvidar el tipo y la valoración de las relaciones interdisciplinarias e 
interinstitucionales. Por último, a modo de conclusión, se debían establecer diferencias basadas en la determinación de objetivos claros y de los niveles de prevención en los que se debería actuar, fundamentado desde un marco teórico específico, a través del uso de la bibliografía incluida en cada módulo.

Como segunda opción metodológica, se ofreció el modelo clásico de presentación de trabajos que incluyera los siguientes puntos: resumen (motivo del trabajo, objetivos, lugar y período temporal en el que fuera realizado, diagnóstico y metodología, resultados y conclusión), introducción, material, métodos (presentación del caso, anamnesis del caso, método de análisis y estudio), resultados (estrategias de intervención, evolución y seguimiento), conclusiones y reflexión (aciertos, fracasos y sugerencias para mejorar la tarea investigativa y de intervención). Todo ello estuvo fundamentado por aportes bibliográficos teóricos de otros autores y especialistas que se vería reflejado en el listado de referencias bibliográficas citadas.

Fue notable entre los participantes el predomino femenino (solo dos varones inscriptos, quienes no presentaron continuidad en el dictado del curso) (figura 6) y el interés manifiesto por los conocimientos recientemente desarrollados por las neurociencias en relación con los indicadores de síndrome de estrés postraumático, compatible con abuso físico, sexual y negligencia en neuroimágenes y marcadores biológicos, a pesar de que la mayoría provenían de disciplinas no médicas.

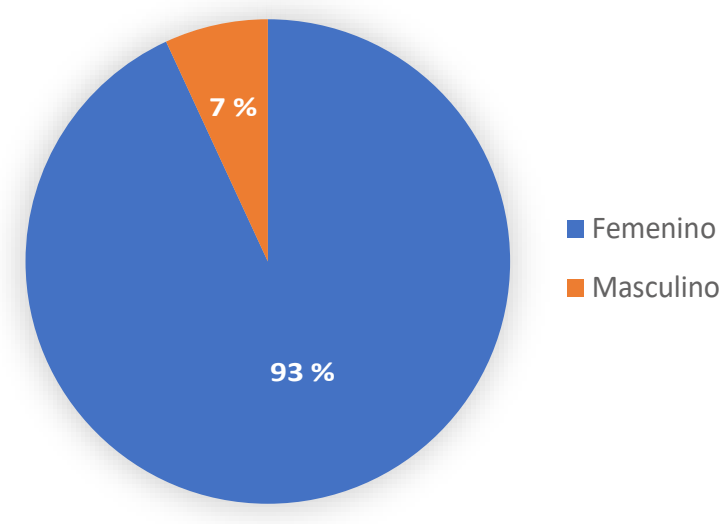

Figura 6. Distribución de estudiantes según el sexo Fuente: elaboración propia.

Las participantes del curso tuvieron la oportunidad, reiteradamente, de expresar sus opiniones acerca de los contenidos conceptuales transmitidos en cada clase, así como sobre las estrategias pedagógicas y técnicas didácticas empleadas. No obstante, finalizado el primer encuentro, surgió un conflicto institucional a 
partir de una versión anónima que descalificaba el rol docente de la profesional que dictaba el curso, por lo que se consultó a los asistentes, quienes manifestaron su disconformidad con esta apreciación subjetiva y mostraron el deseo de continuar con las actividades propuestas. Este hecho podría interpretarse como marcada resistencia a enfrentar los temas relacionados con la violencia que victimiza a los NNA, lo cual quedó más fuertemente demostrado ante el brusco abandono del curso por parte de la estudiante que generó la queja y el conflicto.

$\mathrm{Al}$ acercarse el final del curso, se observó una participación activa del reducido grupo de alumnas que continuaron asistiendo, en el número total de siete (figura 7), cuya participación se manifestó a través del intercambio permanente de experiencias y aportes profesionales acompañados de expresiones de frustración y deseos de cumplimiento de sus ideales.

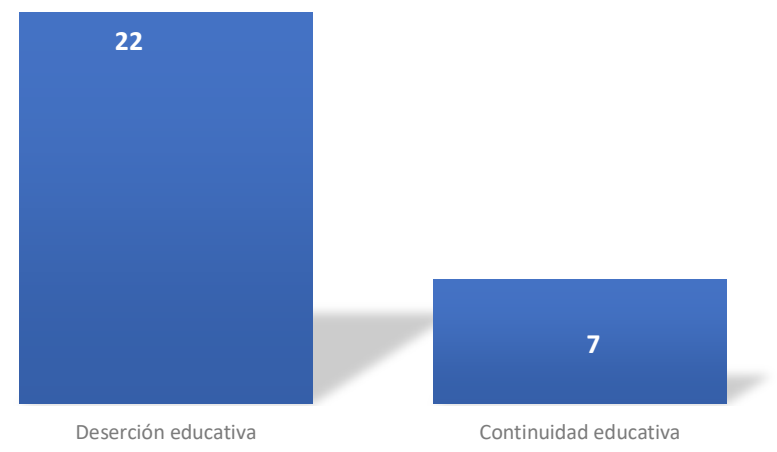

Figura 7. Nivel de continuidad de asistencia de los estudiantes

Fuente: elaboración propia.

Asimismo, las alumnas realizaron interconsultas y derivaciones de casos desde sus lugares de origen. Las asistentes del curso reconocieron y valoraron positivamente los aportes visuales y dinámicos provistos por el material de video de origen foráneo (la presentación con títeres de las reacciones infantiles frente al abuso sexual en $E l$ árbol de Chicoca de origen mejicano; la presentación del tema del abuso físico infantil de acuerdo a la realidad británica, expuesta por la doctora Margaret Lynch, quien es médica pediatra de la Universidad del Este de Londres; el testimonio de los niños víctimas en las Cortes Británicas). Ante la visión de este último video, las participantes pudieron expresar su angustia asociada a la identificación de los sentimientos de temor y vergüenza de las víctimas infantiles durante sus testimonios en la cortes Británicas, mediante la implementación de sistema cerrado de video y, al mismo tiempo, la expresión de sentimientos de admiración por la capacidad adaptativa de los magistrados para comunicarse con los niños testigos, a la vez que surgían sentimientos manifiestos de rechazo por las actitudes intimidatorias de otros letrados. 


\section{Conclusiones}

Frente a los resultados observados, se puede concluir que los profesionales asistentes al curso, en relación con la problemática de victimización infantojuvenil, presentaron un marcado interés por la capacitación y la formación permanente que les permitiera adquirir competencias necesarias, no solo para la interacción con los menores víctimas y sus contextos, sino también para el trabajo interdisciplinario e interinstitucional.

Sin embargo, se pudo comprobar la escasa formación en metodología de la investigación y en el desarrollo de textos científicos, por lo que se recomienda incluir contenidos conceptuales y procedimentales que favorezcan el desarrollo de habilidades investigadoras.

Además, ha resultado muy llamativo el elevado porcentaje de estudiantes femeninas con respecto a los únicos dos varones inscriptos, que tampoco lograron dar continuidad a la actividad formativa y de entrenamiento profesional en esta problemática tan compleja que afecta a la población de niños, niñas y adolescentes, especialmente vulnerables. Esto se podría interpretar como preservación identificatoria cultural de modelos profesionales basados en roles estereotipados de género, en relación con las tareas ancestralmente asignadas a las mujeres con respecto al cuidado de los niños. Teniendo en cuenta esta observación, se deben crear estrategias innovadoras que motiven el interés de los varones por la capacitación y el entrenamiento especializado en problemáticas que afectan a las poblaciones infantojuveniles vulnerables.

Por otro lado, se evidenció notable dificultad para involucrarse emocionalmente en esta problemática por parte de los profesionales, lo cual quedó demostrado por el bajo porcentaje ( $25 \%$ ) de estudiantes que alcanzaron a cumplir los objetivos propuestos, así como por los obstáculos individuales para desarrollar el trabajo grupal. Esto quiere decir que los contenidos relacionados con el sufrimiento infantil por victimización podrían influir negativamente y generar resistencias en los profesionales que se desempeñan en este tipo de problemáticas. Estas mismas resistencias estarían asociadas a los obstáculos institucionales y legislativos que interfieren en el adecuado accionar para defender los derechos de los menores víctimas, lo que conduciría al desarrollo de trastorno de burnt out (síndrome del quemado).

Además, una conclusión de la presente investigación podría ser la elaboración de una teoría innovadora con respecto a las diferentes valoraciones subjetivas individuales sobre la problemática de victimización infantil, que han sido causa de los resultados observados. Estas valoraciones podrían relacionarse, desde el punto de vista fenomenológico, a través de las narrativas de 
los participantes (experiencias de vida que motivan su perfil de personalidad y sus conductas) y de los rasgos de la cohorte de dichos profesionales en estudio. Todo ello podría tender a favorecer prácticas pedagógicas futuras, basadas en la evidencia, que faciliten la adquisición de competencias necesarias para optimizar la resolución de conflictos en victimización infantil teniendo en cuenta el superior interés de NNA.

Para finalizar, se fundamenta la necesidad de favorecer la elaboración de investigaciones educativas debido a que "el conocimiento de lo fáctico necesita registrarse para beneficio de las generaciones futuras, que lo pondrán a prueba de sus dudas y sus nuevas hipótesis relacionadas con sus ambiciosos deseos" (Martorella, 2013a, p. 2).

\section{Agradecimientos}

Agradezco a la profesora Anna Candreva, por su generosidad y competencias en la transmisión de saberes y experiencia profesional en pedagogía médica; a mis alumnos y alumnas comprometidos con la adquisición de habilidades profesionales para la intervención adecuada en los casos de infancias vulnerables: a mi esposo y compañero de sueños y utopías; a los revisores de mi trabajo, por sus sugerencias y señalamientos; a los editores de la revista, especialmente Sebastián Suaza Palacio y al doctor Hilderman Cardona Rodas, por su interés por este tipo de problemáticas y su enseñanza a profesionales.

Cabe destacar que este trabajo surge como resultado de una tarea de investigación educativa propuesta durante el curso de Pedagogía Médica del Plan de Formación Docente Continuo, dirigido por la profesora doctora Anna Candreva en el Colegio de Médicos de la provincia de Buenos Aires (distrito IX), por convenio con el Departamento de Pedagogía Médica de la Universidad Nacional de La Plata, en el ciclo lectivo 2002, presentado con el título de Curso: ateneos clínicos en abuso infantil y negligencia.

Además, es el resultado de otro trabajo presentado en el ciclo lectivo 2005 en el módulo Pedagogía médica: rol de los tutores e investigación educativa, dictado por la misma profesora, cuyo título es Investigación educativa: prevención de abuso infantil y negligencia. Este fue presentado como comunicación científica, junto a la doctora Angela Lida Calderaro (coautora) en el XXII Congreso Argentino de Psiquiatría de APSA, llevado a cabo en la ciudad de Mar del Plata, del 27 al 30 de abril de 2006, con el título Resultados de un entrenamiento en prevención de abuso infantil. 


\section{Referencias}

Bélisle, C. y Linard, M. (1996). Quelles nouvelles compétences des acteurs de la formation dans le contexte des TIC? Éducation Permanente (127), 19-47.

Brassard, M. y Hardy, D. (1984). Psychological Maltreatment, The Battered Child. Fifth edition. The University of Chicago Press.

Boyatzis, R. (1982). The competent manager. A model for effective performance. Wiley \& Sons.

Bunk, G. (1994). La transmisión de competencias en la formación y perfeccionamiento profesionales en la RFA. Revista Europea de Formación Profesional, 1, 8-14. https://dialnet.unirioja.es/servlet/ articulo?codigo $=131116$

Candreva, A., Martorella, A., y Susacasa, S. (2011). Competencias vinculadas a la psiquiatría en el ejercicio general y de las especialidades. Educación Médica Permanente, 2(2), 36-37.

Gilbert, P. y Parlier, M. (1992). La compétence: du "mot valise" au concept opératoire. Actualité de la formation permanente, 116, 14-18. https://www.researchgate.net/publication/271199193_La_competence_Du_mot_valise_au_concept_operatoire

Ginisty, D. (1997). L'home au centre du debat sur les competences, Entreprisesformation, 103, 16-17.

Gobierno de Canadá (2007). Key Leadership Competencies, Canada Public Service Agency and the Public Service Commission.

González, N., Zerpa, M., Gutierrez, D. y Pirela, C. (2007). La investigación educativa en el hacer docente. Laurus, 13(23), 279-309. https://www.redalyc.org/pdf/761/76102315.pdf

Hayes, R. H. (1985). Strategic Planning Forward in Reverse. Harvard Business Review, 111-119. https:// hbr.org/1985/11/strategic-planning-forward-in-reverse

Helfer, M., Kempe, R. y Krugman, R. (1984). The Battered Child (5a ed). The University of Chicago Press.

Hume, D. (1984). Tratado de la naturaleza Humana. Ediciones Orbis.

Jessup, G. (1991). Outcomes: NVQs and the emerging model of education and training. Falmer Press.

Klimovsky, G. (1994). Las desventuras del conocimiento científico: Una introducción a la epistemología. A-Z Editora.

Laudan, L. (1986). El progreso y sus problemas. Hacia una teoría del crecimiento científico. Ediciones Encuentro.

Le Boterf, G. (1994). De la competence. Les Editions d'Organisations.

Le Boterf, G. (1997). Entrevista por Aattane, CH. Entreprises-formation, 100, 48-49.

Le Boterf, G. (2000). Construire les competentes individuelles et collectives. Editions d'Organisation.

Losada, A. V. (2012). Epidemiología del abuso sexual infantil. Revista de Psicología GEPU, 3(1), 201-229

Malpica, M. (1996). El punto de vista pedagógico. En A. Argüelles (ed.), Competencia laboral y educación basada en normas de competencia (pp. 123 -140). Limusa.

Martorella, A. y Portugués, A. (1998). Prevention of Sexual Abuse in Children with Learning Disabilities. Child Abuse Review, 7 (5), 355-359. https://doi.org/10.1002/(SICI)10990852(199809/10)7:5\%3C355::AID-CAR506\%3E3.0.CO;2-O 
Martorella, A. (2010). Abuso sexual infantil: El juego como factor de resiliencia [conferencia]. XI Congreso Virtual Internacional de Psiquiatría, Psicología y Salud Mental (Interpsiquis). http://psiqu.com/1-998

Martorella, A. y Candreva, A. (2011). Competencias de Psiquiatría necesarias para el ejercicio profesional de otras especialidades médicas. [conferencia]. XII Congreso Virtual Internacional de Psiquiatría, Psicología y Salud Mental (Interpsiquis). https://bit.ly/2OppgCx

Martorella, A. (2013a). Construcción Histórica del conocimiento científico. Psiquiatría.com. https://psiquiatria.com/bibliopsiquis/construccion-historica-del-conocimiento-cientifico

Martorella, A. (2013b). El Rol del Médico en la Educación sexual Infantil y del Adolescente. Tercera Época. Revista de la Facultad de Ciencias Médicas, 4(2), 1-1. http://revista.med.unlp.edu.ar/archivos/201312/27-Martorella.pdf

Martorella, A. (2014a). Educación Sexual para Alumnos con Retardo Mental Leve. [conferencia]. XV Congreso Virtual Internacional de Psiquiatría, Psicología y Salud Mental (Interpsiquis). http:// psiqu.com/1-5255

Martorella, A. (2014b). La educación sexual y el sistema educativo. XV Congreso Virtual Internacional de Psiquiatría, Psicología y Salud Mental (Interpsiquis). http://psiqu.com/1-5254

Martorella, A. (2015). Revictimización de los niños víctimas de abuso sexual infantil intrafamiliar por el poder judicial. Bibliopsiquis. Blogs de psiquiatría. http://psiqu.com/1-1874

Montmollin, P. (1984). L'intelligence de la tache. Lang.

Perrenoud, P. (1999). Construir competencias desde la escuela. Dolmen.

Sainz Leyva, L. (2002). La evaluación del aprendizaje: Educación vs. Autoridad: una propuesta formativa y no impositiva en pos del consenso profesor-estudiante. Revista Electrónica Interuniversitaria de Formación del Profesorado, 5(1). https://dialnet.unirioja.es/servlet/articulo?codigo=1031447 